\section{Schwannoma en el puerperio}

\author{
Schwannoma in the puerperium
}

\author{
Dra. Daria Irina Romero-Luna,* Dr. Arturo Jaime Córdova, ${ }^{\ddagger}$ \\ Dra. Yasiu Bustamante-Quan, ${ }^{\S}$ Dra. Yolanda Valera-Rodríguez, ${ }^{\natural}$ \\ Dr. Alberto Kably-Ambell
}

Citar como: Romero-Luna DI, Córdova AJ, Bustamante-Quan Y, Valera-Rodríguez Y, Kably-Ambe A. Schwannoma en el puerperio. Rev Mex Anestesiol. 2022; 45 (2): 142-144. https://dx.doi.org/10.35366/103891

\begin{abstract}
RESUMEN. Se presenta el caso de una paciente de 48 años, previamente sana, sometida a cesárea bajo bloqueo epidural que presenta datos de compresión medular en el puerperio inmediato. El diagnóstico inicial fue una complicación por anestesia neuroaxial, después de la resonancia magnética se reporta tumor intrarraquídeo a nivel de T9-T10 que fue extirpado con diagnóstico histológico de schwannoma. La evolución postquirúrgica de la paciente fue favorable sin secuelas neurológicas. Los schwannomas son tumores benignos cuya principal manifestación son síntomas de compresión medular.
\end{abstract}

ABSTRACT. It is presented a case of a 48-year-old patient, previously healthy, who underwent cesarean section under epidural block and presented data of spinal cord compression in the immediate puerperium. The initial diagnosis was a complication due to the neuraxial anesthesia. Afterwards, the MRI reported an intraspinal tumor at the T9-T10 level, which was removed with a histological diagnosis of schwannoma. The patient's postoperative evolution was favorable, with no neurological sequelae. Schwannomas are benign tumors, whose main manifestation is symptoms of spinal cord compression.

\section{INTRODUCCIÓN}

T. a presencia de un tumor en el embarazo es rara, ocurre en uno de cada 1,000 a 2,000 embarazos, siendo los más frecuentes el de mama, hematológicos y dermatológicos ${ }^{(1)}$.

Los tumores del sistema nervioso central comprenden un grupo heterogéneo de neoplasias que tiene una gran diversidad histológica y están confinados a cerebro, médula espinal, meninges, nervios craneales y paraespinales. Su incidencia se ha reportado de 6.3 casos por $10^{5}$ habitantes en países desarrollados y de 9.9 por $10^{5}$ habitantes en países en vías de desarrollo ${ }^{(2,3)}$.

Un estudio realizado por Anaya y colaboradores acerca de la prevalencia de los tumores del sistema nervioso central en población mexicana, encontró que los tumores de meninges fueron los más frecuentes (33\%), seguidos de los de neuroepitelio (24\%), región selar (19\%) y nervios craneales y paraespinales (13\%). Dentro de los tumores de nervios craneales y paraespinales el schwannoma ocurre en $10.37 \%$ y el neurofibroma en $2.34 \%{ }^{(2)}$.

Los schwannomas son tumores benignos de crecimiento lento, en forma de «pera», cuya localización periférica más frecuente es a nivel de la columna dorsal y raramente malignizan. Representan el 25\% de los tumores intradurales en adultos y se calcula que el diagnóstico tarda aproximadamente $6.7 \pm 2$ años con una progresión clínica crónica. La sintomatología más común es dolor neuropático, exacerbado por el movimiento, ello por su predilección por las fibras sensitivas sobre las motoras. En los casos en los que se presenta un crecimiento acelerado, éste se acompaña de compresión medular con mielopatía. El pico de incidencia ocurre entre la tercera y la sexta década de la vida, como el caso de la paciente, sin predilección étnica ni de género ${ }^{(3,4)}$.

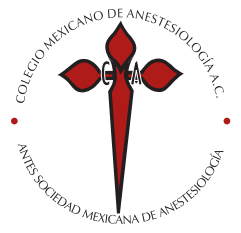

Palabras clave:

Schwannoma, bloqueo epidural, analgesia postquirúrgica.

\section{Keywords:}

Schwannoma, epidural block, postsurgical analgesia.

* Médico Anestesióloga, Hospital ABC Campus Santa Fe. Hospital General de Zona No. 98, IMSS.

San Francisco Coacalco. México.

‡ Médico Anestesiólogo, Hospital ABC Campus Santa Fe. Hospital General Regional No. 196, IMSS. Ecatepec de Morelos. México.

§ Médico Ginecóloga.

` Médico Anestesióloga, Hospital General Regional No. 196, IMSS. Ecatepec de Morelos. México.

" Ginecología y Obstetricia, Subespecialidad en Biología de la Reproducción Humana, Director del Centro Mexicano de Fertilidad «Dr. Alberto Kably». Valle de las Palmas. México.

Hospital Ángeles Lomas. Estado de México. México.

Correspondencia:

Dra. Daria Irina Romero-Luna Hospital Ángeles Lomas, Vialidad de la Barranca s/n, Huixquilucan, Estado de México. E-mail: daris_lala@hotmail.com

Recibido: 13-03-2020 Aceptado: 15-10-2020 
En 2015 Meng informó sobre una serie de casos de embarazadas con tumores espinales, en las cuales el diagnóstico se realizó principalmente en el segundo trimestre de sus embarazos. En esta serie los tipos de tumor más frecuentes consistieron en tumores de células gigantes (cinco casos), hemangioma (cinco casos) y schwannomas (cuatro casos). La sintomatología en estos últimos fue de hipoestesias, dolor, debilidad y el hallazgo del tumor. En todos los casos de schwannoma el tratamiento consistió en controlar el dolor con analgésicos y extirpación del tumor posterior al parto y cuando causaba incapacidad funcional ${ }^{(1)}$.

\section{CASO CLÍNICO}

Paciente de 48 años en su primera gestación, ingresada para la resolución de su embarazo vía cesárea tipo Kerr a las 38.0 semanas de gestación ante producto por fertilización in vitro. La paciente refirió la presencia de dolor lumbar bajo con irradiación a miembros pélvicos con sensación de ardor, interpretado como neuritis lumbociática. Su presentación era ocasional y no invalidante que cedía al reposo; por tanto, no recibió tratamiento analgésico. Ante esta dolencia, se le realizó una resonancia magnética nuclear (RMN) lumbar con reporte de estudio sin ninguna patología. A la exploración durante el internamiento obstétrico no se evidenciaron alteraciones anatómicas ni dolor en la región vertebral. La valoración neurológica fue normal. En conjunto con estudios de laboratorio normales se le clasificó con un riesgo anestésico ASA I. Por lo tanto, se decidió realizar anestesia neuroaxial con monitoreo tipo I, bloqueo epidural a nivel L2-L3 con aguja Tuohy \# 16 con técnica de pérdida de la resistencia y dosis de prueba sin incidentes. El bloqueo se realizó con dosis fraccionada de lidocaína al $2 \%$ con epinefrina, la mitad a través de la aguja y el resto a través de un catéter epidural cefálico previamente colocado. Además, se infundió fentanilo $(100 \mu \mathrm{g})$ como coadyuvante. La cesárea se realizó sin ningún incidente, con un sangrado calculado de un aproximado de $300 \mathrm{~mL}$.

Durante la hora de estancia en la Unidad de Cuidados Postanestésicos, se comprueba la recuperación anestésica con un puntaje Aldrete de 9 y un Bromage de 1. Ante la recuperación, es enviada a piso de obstetricia para vigilancia, con analgesia regional postquirúrgica con bomba PCA (Pain Control Analgesia) con ropivacaína al 7.5\% (150 mg) más fentanilo $(300 \mu \mathrm{g})$ aforado a $120 \mathrm{~mL}$ de solución salina $0.9 \%$, para infusión a $3.5 \mathrm{~mL} / \mathrm{h}$, con dosis de rescate de $2.5 \mathrm{~mL} \mathrm{c} / 4$ h. En la evaluación del dolor por ENA (Escala Numérica Análoga), la paciente manifestó ausencia de dolor, pero sensación de parestesia a nivel de dermatomas T12-S5 durante las siguientes 12 horas postcirugía.

A partir de las 12 horas de inicio de bomba PCA, la paciente manifiesta ausencia de movimiento en ambos miembros inferiores. En la valoración por anestesiología se confirma datos de bloqueo motor, por lo cual se retira el catéter epidural y la analgesia postquirúrgica. La paciente es valorada por el servicio de neurología, quienes confirman la presencia de hiperreflexia $4 / 4$ en cuádriceps bilateral y $2 / 4$ en aquiliano bilateral, aunado con Babinski bilateral. Además de vibración disminuida desde tercio inferior de ambos miembros, pero con la conservación de la posición articular, termocepción y propiocepción.

A la paciente se le realiza una RMN de columna toraco lumbo sacra, simple y con gadolinio ante la sospecha de una compresión medular a nivel de T10-11. Las imágenes evidenciaron un tumor intrarraquídeo extramedular en su parte anterior localizado entre los segmentos T9-T10, el cual comprimía la médula en sentido anteroposterior.

Se inició manejo con esteroides para prevención de choque medular con dexametasona $8 \mathrm{mg} / 8 \mathrm{~h} \mathrm{IV}$.

La paciente fue sometida a resección tumoral a través de laminectomía a nivel T9-T11. El procedimiento fue realizado sin incidentes y se resecó sin problemas una masa tumoral de $2.8 \times 1.0 \times 0.8 \mathrm{~cm}$ en sus diversos diámetros. El reporte histopatológico transquirúrgico fue de schwannoma.

El postoperatorio a esta segunda intervención cursó sin complicaciones. Se continuó manejo con esteroide por cinco días más. La paciente recuperó la movilidad voluntaria con fuerza muscular $5 / 5$ y sin alteraciones sensitivas a partir de las 48 horas postcirugía. Fue egresada a su domicilio caminando al séptimo día posterior a manejo de rehabilitación.

\section{DISCUSIÓN}

El objetivo de presentar este caso fue exponer nuestra experiencia sorpresiva de una sintomatología de bloqueo motor posterior a una anestesia neuroaxial exitosa sin incidentes ni accidentes y con recuperación de la sensibilidad y movimientos en el postanestésico inmediato.

Ante este comportamiento agudo, las posibilidades diagnósticas consideradas fueron un hematoma epidural o un síndrome de la arteria espinal que pueden causar paraplejia irreversible $^{(5)}$. Estas posibilidades fueron desestimadas ante la ausencia de incidentes o accidentes durante la técnica del bloqueo de la paciente. Además, la presentación de los síntomas ante estas complicaciones suele ocurrir a las pocas horas después del procedimiento anestésico y no a las 12 horas posteriores como en nuestra paciente. Otra posibilidad remota considerada fue un efecto acumulativo de la analgesia durante el manejo de PCA. Ante la inquietud se decidió retirar la misma. Aunque esta complicación es extremadamente rara.

Finalmente, ante la persistencia del compromiso motor con datos de neurona motora inferior, se decidió realizar una RMN. En esta ocasión, ante una exploración más dirigida, se decidió incluir la sección torácica. Esto permitió evidenciar el tumor que estaba comprimiendo la médula espinal. Un 
tumor en el canal medular en el embarazo puede ser de difícil diagnóstico para un médico, ya que los síntomas pueden confundirse con los cambios esperados en la gestación, tales como: dolor lumbar o parestesias en extremidades. Es de llamar la atención que en esta paciente la molestia fue tan recurrente que motivó un estudio de RMN. El principal error fue la equivocación en la exploración por imagen de la zona afectada. De haberse realizado un estudio más amplio el tumor hubiera sido detectado y manejado previamente.

Con respecto a los schwannomas, por su hipervascularidad, edema y los cambios hemodinámicos del embarazo, algunos tumores pueden necrosarse o tener hemorragias espontáneas y con ello causar compresión por el aumento de la presión en el espacio epidural secundaria a cambios fisiológicos del embarazo $^{(1,3,6,7)}$. Sin embargo, en nuestra paciente el tumor no parece haber progresado o complicado ante la ausencia de síntomas en los días previos a la cesárea.

Consideramos que la presentación de la sintomatología en el postoperatorio mediato pudo deberse a cambios constantes de la presión a nivel del espacio epidural. Normalmente el espacio epidural tiene una presión de $-5 \mathrm{~cm}^{3}$ de $\mathrm{H}_{2} \mathrm{O}$, por lo cual la aplicación de un volumen de $10 \mathrm{~cm}^{3}$ sobre este espacio provocaría un aumento de hasta $60 \mathrm{~cm}$ de $\mathrm{H}_{2} \mathrm{O}$ en el mismo. Estudios previos han demostrado que esta presión disminuye en los siguientes 30 segundos, pero dejando siempre una presión residual dentro del espacio ${ }^{(7,8)}$. Esta presión en condiciones habituales no causaría problema, pero en nuestra paciente con un tumor extramedular la presión sería más persistente $^{(5,9)}$. Esto es, la aplicación de la anestesia neuroaxial aumentó la presión en el espacio epidural, persistiendo una presión residual, la cual se incrementó por el uso de la infusión continua de la PCA.

\section{CONCLUSIONES}

Los tumores en el embarazo tienen una baja incidencia, en especial los localizados en la médula espinal. Los cambios adaptativos de la gestación pueden simular varias patologías y dificultar diagnósticos como éstos. Ante una evolución atípica, la revaloración y el manejo interdisciplinario con prontitud pueden resolver problemas previamente no detectados.

\section{REFERENCIAS}

1. Meng T, Yin H, Li Z, et al. Therapeutic Strategy and Outcome of Spine Tumors in Pregnancy. Spine. 2015;40: E146-E153.

2. Anaya G, de Juambelz PP, Fernández B, et al .prevalencia de tumores del sistema nervioso central y su identificación histológica en pacientes operados: 20 años de experiencia. Cirugía y Cirujanos. 2016;84:447-453.

3. Chen R, Xiao A, Xing L, et al. A rare thoracic intraspinal schwannoma in twin pregnancy with aggravated clinical presence. Medicine. 2017;96:1-3.

4. Serrano A, Orenday J, Arriada N. Análisis de la resección de schwannomas espinales experiencia en una sola institución. Arch Neurocien 2017;22:31-27.

5. Whizar V, Flores J. Complicaciones neurologicas en la paciente obstetrica sometida a Anestesia Neuroaxial. Anestesia obstrética. 2008;379-398. Disponible en: www.anestesia-dolor.org
6. Pérez-Carbajo E, Zapardiel-Gutiérrez I, Frutos-LLorente L, et al. Schwannoma en espina dorsal en gestante en 24 semanas. Prog Obstet Ginecol. 2011;54:21-23.

7. Karatay M, Kokterir E, Erdem Y, et al. Intramedullary schwannoma of conus medullaris with syringomyelia. Asian Journal of Surgery. 2017;40:240-242.

8. Usubiaga JE, Wikinski JA, Usubiaga LE. Epidural pressure and its relation to spread of anesthetic solutions in epidural Space. Anesth Analg. 1967;46:440-446.

9. Hussain A, Bhakta P, Singh V. Anesthetic management of cesarean section in patient complicated with neuroectodermal tumor: a case report. J Clin Anesth. 2015;27:532-533. 